\title{
Background Factors Determining the Introduction and Dosage of Insulin in Women With Gestational Diabetes Mellitus
}

\author{
Yuko Matsumoto a, Hodaka Yamada ${ }^{\mathrm{a}, \mathrm{c}}$, Masashi Yoshida ${ }^{\mathrm{a}}$, Daisuke Suzukia, \\ Rika Saikawa $^{a}$, Misato Amamoto ${ }^{a}$, Shunsuke Funazakia ${ }^{a}$, Isao Horiuchi ${ }^{b}$, \\ Kenjiro Takagi ${ }^{\text {, }}$ Kazuo Hara ${ }^{a}$
}

\begin{abstract}
Background: Gestational diabetes mellitus (GDM) is a risk for perinatal complication, and appropriate diagnosis of and intervention in this condition are important. This study aimed to identify patient factors associated with introduction and dosage of insulin, which is the main drug for treatment of GDM.

Methods: In total, 114 patients who had been diagnosed with GDM at our hospital were included in this study. We retrospectively collected clinical parameters of GDM patients, including how many times positive glucose tolerance test results were obtained, whether insulin was introduced, dosage of insulin, body weight, and infant weight. Background factors differing between the insulin introduction and non-introduction groups of GDM patients and parameters associated with the insulin dosage were analyzed.
\end{abstract}

Results: Insulin was introduced in 51 GDM patients (45\%). In the insulin introduction group, the six-divided diet was less common and the 75-g glucose tolerance test result was positive a significantly greater number of times compared with the non-introduction group. The factor associated with the insulin introduction status was the number of positive $75-\mathrm{g}$ glucose tolerance test results (odds ratio (OR) 2.04, 95\% confidence interval (CI): 1.09 - 3.81, $\mathrm{P}$ value $=$ $0.025)$. In addition, the insulin dosage was found to positively correlate with body weight in the non-pregnant state $(\mathrm{P}$ value $=0.005)$.

Conclusions: The six-divided diet was effective for blood glucose

Manuscript submitted March 22, 2019, accepted April 16, 2019

aDepartment of Medicine, Division of Endocrinology and Metabolism, Jichi Medical University Saitama Medical Center, 1-847 Amanuma-cho, Omiya-ku, Saitama 330-8503, Japan

bPerinatal and Maternal Center of Saitama Medical Center, Jichi Medical University Saitama Medical Center, 1-847 Amanuma-cho, Omiya-ku, Saitama 330-8503, Japan

${ }^{\mathrm{c}}$ Corresponding Author: Hodaka Yamada, Department of Medicine, Division of Endocrinology and Metabolism, Jichi Medical University Saitama Medical Center, 1-847 Amanuma-cho, Omiya-ku, Saitama 330-8503, Japan.

Email: hyamada0510@jichi.ac.jp

doi: https://doi.org/10.14740/jocmr3824 control in GDM women. A positive correlation found between the insulin dosage and body weight in the non-pregnant state suggests the importance of proper pre-pregnancy body weight control.

Keywords: Gestational diabetes mellitus; Diet therapy; Insulin therapy; Obesity

\section{Introduction}

Gestational diabetes mellitus (GDM) can cause fetal hyperglycemia and hyperinsulinemia, resulting in macrosomia or neonatal hypoglycemia [1]. It is also known to be a cause of maternal complications such as pregnancy-induced hypertension and pregnancy wastage [2]. To avoid the risk for these maternal and fetal complications, it is crucial to prevent the onset of GDM or detect and start treatment of GDM at an early stage [3]. After the International Association of Diabetes and Pregnancy Study Group (IADPSG) issued new diagnostic criteria of GDM based on Hyperglycemia and Adverse Pregnancy Outcomes (HAPO) in 2010 [4], the number of GDM patients in Japan has markedly increased compared with that when the conventional criteria were used [5]. The present study aimed to clarify the clinical background, treatment methods (diet therapy and introduction of insulin therapy), and perinatal and postpartum outcomes in GDM patients in our hospital. Predictors of GDM onset and the necessity of insulin treatment identified in this study are expected to contribute to prevent worsening of GDM and maternal and fatal complications through early interventions in GDM as well as provide perinatal care guidance to prevent the onset of GDM; therefore, we believe that this study has an implication for the improvement of maternal and child health.

\section{Materials and Methods}

This is a retrospective, single-center, observational cohort study. This study was approved by the Ethics Committee of the Jichi Medical University, Saitama Medical Center (No. S17- 
003) and conforms to the ethical guidelines of the Declaration of Helsinki. In total, 114 pregnant women who underwent GDM screening and were diagnosed with GDM at our hospital between April 2013 and December 2016 were included in this study, except for those with a complication of diabetes mellitus. For GDM screening, a 50-g glucose challenge test (cut-off value: $140 \mathrm{mg} / \mathrm{dL}$ ) was performed in the second trimester of pregnancy. Women who tested positive in the initial screening test underwent a 75-g oral glucose tolerance test (75-g OGTT), and were diagnosed with GDM if the results meet GDM diagnostic criteria in Guidelines for Obstetrical Practice in Japan 2014 [6].

All women diagnosed with GDM were hospitalized and were fed six-divided diet with tripartition energy (total energy intake (kcal): body mass index (BMI) $<25 \mathrm{~kg} / \mathrm{m}^{2}$, standard body weight $\times 30+250(+50$ in the first trimester $) ; B M I \geq 25$ $\mathrm{kg} / \mathrm{m}^{2}$, standard body weight $\times 30$ according to the Guidelines for the Management of Diabetes Mellitus 2016 [7] under selfmonitoring of blood glucose. The target blood glucose level was to satisfy both $<100 \mathrm{mg} / \mathrm{dL}$ preprandially and $<120 \mathrm{mg} /$ $\mathrm{dL}$ postprandially according to the Guidelines for the Management of Diabetes Mellitus 2016 [7], and the six-divided diet therapy was introduced in the case of poor blood glucose control. Insulin therapy was introduced when the target blood glucose level was not met even after the introduction of the six-divided diet therapy.

\section{Analysis 1: association between the introduction of insulin therapy and background factors in GDM patients}

The following background factors were analyzed to check whether they had any association with the introduction of insulin therapy: age (years), prevalence of family history of impaired glucose tolerance, non-pregnant body weight (kg), body weight at delivery (kg), BMI, Apgar scores at $1 \mathrm{~min}$ and $5 \mathrm{~min}$ (points), newborn weight (g), placental weight (g), introduction of the six-divided diet, the number of positive 75-g OGTT results (positive at one time point vs. positive at two or three time points).

\section{Analysis 2: association between insulin dosage (bolus insu- lin) and background factors in patients for whom insulin was introduced (51 patients)}

The following background factors were analyzed to check whether they had any association with insulin dosage (bolus insulin): age (years), prevalence of family history of impaired glucose tolerance, non-pregnant maternal body weight $(\mathrm{kg})$, placental weight $(\mathrm{g})$, and introduction of the six-divided diet.

\section{Statistical analysis}

Results were represented in the form of mean value \pm standard deviation or median ( 25 - 75 th percentile). Student's $t$-test or U-test was used for comparison between two groups. Fisher's exact test was used for categorical variables. Multiple regression analysis or logistic regression analysis was used for multivariate analysis. Pearson or Spearman correlation analysis was used for correlation analysis. EZR was used for statistical analyses, and $\mathrm{P}<0.05$ was considered to indicate a statistically significant difference.

\section{Results}

In the overall study population, the age was $34.4 \pm 4.5$ years; insulin was introduced in 51 cases $(44.7 \%)$ and not introduced in 63 cases; daily insulin dose was $10 \pm 15.8$ units/day; 48 patients $(42.1 \%)$ had a family history of impaired glucose tolerance and 66 patients did not; newborn weight was 2,761 \pm 446 g; and 66 patients $(57.9 \%)$ underwent six-divided diet therapy and 48 did not. In 75-g OGTT, 80 patients tested positive once, and 34 patients tested positive two or three times (Table 1).

In analysis 1, background factors with differences between the insulin introduction and non-introduction groups were the introduction of the six-divided diet and number of positive 75-g OGTT results (Table 2). No differences were found with respect to age, prevalence of family history, non-pregnant maternal body weight, prevalence of obesity, newborn weight, or placental weight. In addition, logistic regression analyses were conducted to identify clinical background parameters contributing to the introduction of insulin therapy. Of the explanatory variables (prevalence rates of family history, late-in-life pregnancy, and obesity and the number of positive 75-g OGTT results), the number of positive 75-g OGTT results was the only significant explanatory variable (odds ratio (OR) 2.04, $95 \%$ confidence interval $(\mathrm{CI}): 1.09-3.81, \mathrm{P}=0.025)$ (Table 3 ).

In analysis 2 , single and multiple regression analyses were performed, and an association was found between insulin dosage and non-pregnant body weight $(\mathrm{r}=0.330, \mathrm{P}=0.005)(\mathrm{Ta}-$ ble 4). The correlation analysis revealed a positive correlation between the insulin dosage and non-pregnant body weight ( $\mathrm{r}=$ $0.360, P=0.012$ ) (Fig. 1). In addition, the insulin dosage was significantly lower in the group of patients who underwent sixdivided diet therapy $(\mathrm{P}=0.034)$.

\section{Discussion}

A previous study has shown that an increased number of positive 75-g OGTT results was associated with an increased likelihood of the introduction of insulin therapy [8]. In analysis 1 of the present study, we explored other factors for determining the introduction of insulin therapy. The first background factor that differed between the insulin introduction and non-introduction groups was the number of positive 75-g OGTT results, as was the case in the previous study. The present study also showed that the necessity of introducing insulin was predictable with the number of positive 75 -g OGTT results. Starting therapeutic intervention early in the positive 75-g OGTT result group with obesity, which is a factor requiring insulin treatment identified in a previous study [9], appears to be of particular importance. The presence of obesity was not a risk 
Table 1. Patient Background

\begin{tabular}{ll}
\hline Item & Mean \pm SD \\
\hline Age (years) & $34.4 \pm 4.5$ \\
Insulin introduction (with/without) & $51(45 \%) / 63(55 \%)$ \\
Bolus insulin used (units/day) & $10 \pm 15.8$ \\
Family history of diabetes mellitus (with/without) & $48(42 \%) / 66(58 \%)$ \\
Non-pregnancy body weight (kg) & $60.1 \pm 12.6$ \\
Number of weeks of pregnancy at the time of diagnosis (weeks) & $22 \pm 6$ \\
Body weight at the time of delivery (kg) & $66.3 \pm 12.0$ \\
Apgar score at 1 min & $7.89 \pm 0.71$ \\
Apgar score at 5 min & $8.87 \pm 0.56$ \\
Newborn weight (g) & $2,761 \pm 446$ \\
Placental weight (g) & $585 \pm 177$ \\
Six-divided diet (with/without) & $66(58 \%) / 48(42 \%)$ \\
Number of positive 75 -g OGTT results (once vs. twice or thrice) & $80 / 34$ \\
\hline
\end{tabular}

75-g OGTT: 75-g oral glucose tolerance test.

factor for insulin introduction identified in analysis 1. In Japan, $14.3 \%$ of women in their 30 s are obese [10]; however, the prevalence of obesity in this study was $38.6 \%$. The higher prevalence is likely because our hospital is a university hospital and has many patients with the obesity complication. A future study with an increased number of patients is necessary.

The second risk for insulin introduction was the six-divided diet; significantly more patients in the insulin introduction group underwent the six-divided diet therapy. In our hospital, patients diagnosed with GDM are hospitalized as a rule, fed with the six-divided diet with tripartition calories according to the Guidelines for the Management of Diabetes Mellitus 2016 [6], and have the blood glucose level measured before and $2 \mathrm{~h}$ after each meal. The six-divided diet therapy was introduced if patient's preprandial and 2-h-postprandial blood glucose levels were $\geq 100 \mathrm{mg} / \mathrm{dL}$ and $\geq 120 \mathrm{mg} / \mathrm{dL}$, respectively, for at least 2 days. This is presumably why significantly more patients in the insulin introduction group underwent six-divided diet therapy. Analysis 2 explored factors related to insulin dosage in the insulin introduction cases. The first factor identified was the introduction of the six-divided diet. The insulin dosage in the six-divided diet introduction group was smaller than that in the non-introduction group. Horie et al have reported that diet therapy improved glucose tolerance and no longer met GDM criteria in the second trimester in 18 of 41 pregnant women who were found to have impaired glucose tolerance in the first trimester [11]. Another study has shown that diet therapy could delay the time of insulin introduction [12]. These findings indicate that the introduction of the sixdivided diet is useful for blood glucose control. It is likely that patients with positive 75-g OGTT results benefit from the introduction of diet therapy. In our hospital, nutritionists provide both inpatients and outpatients with nutritional guidance, and pregnant women self-check if the energy intake level is

Table 2. Background Factors That Differed Between the Insulin Non-Introduction Group and the Insulin Introduction Group

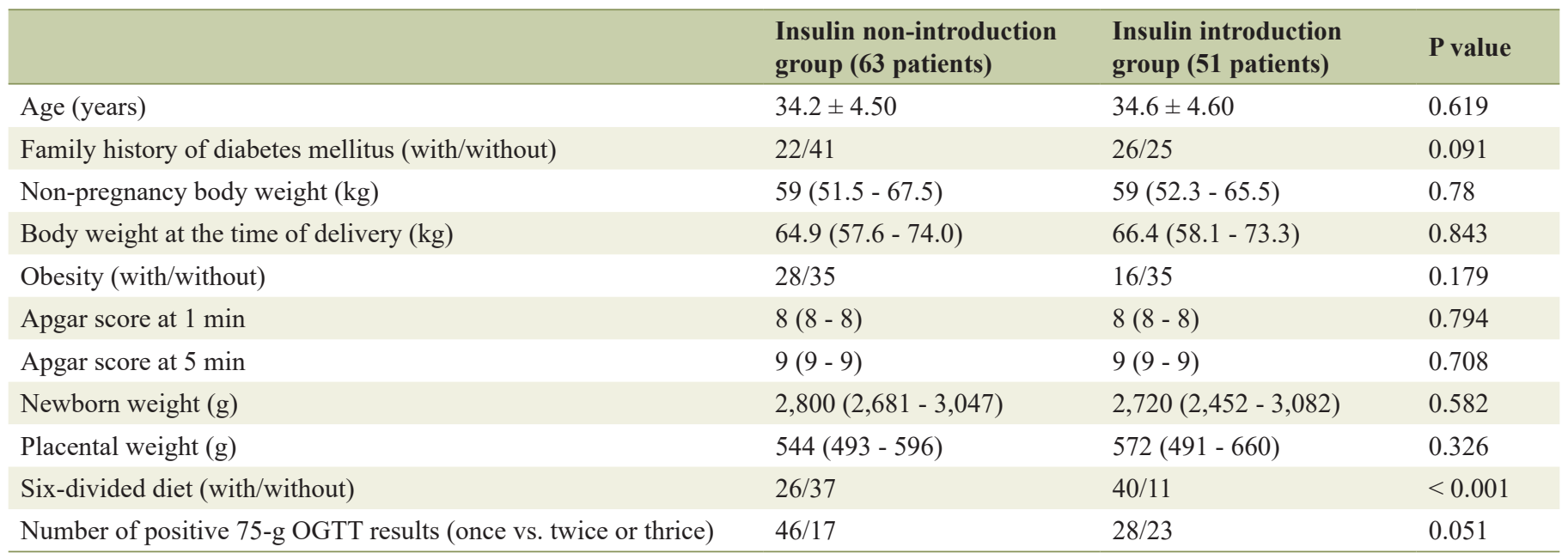


Table 3. Background Factors Contributing to the Necessity of Insulin Introduction

\begin{tabular}{llll}
\hline Univariate analysis & & & P value \\
\hline With/without family history & OR & $\mathbf{9 5 \% ~ C I ~}$ & 0.086 \\
With/without late-in-life pregnancy & 1.94 & $0.91-4.12$ & 0.616 \\
With/without obesity & 1.02 & $0.94-1.11$ & 0.325 \\
Number of positive 75-g OGTT results & 1.04 & $0.96-1.12$ & 0.026 \\
\hline Multivariate analysis & 2.04 & $1.09-3.81$ & P value \\
\hline
\end{tabular}

Table 4. Insulin Dosage and Background Factors

\begin{tabular}{llll}
\hline Univariate analysis & & & P value \\
\hline Age (years) & Estimate & Std. Er & 0.500 \\
Body weight at the time of delivery & 0.224 & 0.331 & 0.007 \\
Non-pregnancy body weight $(\mathrm{kg})$ & 0.33 & 0.120 & 0.114 \\
Placental weight $(\mathrm{g})$ & 0.33 & 0.01 & 0.164 \\
\hline Multivariate analysis & 0.014 & & P value \\
\hline Non-pregnancy body weight $(\mathrm{kg})$ & & Std. Er & 0.005 \\
\hline
\end{tabular}

Std. Er: standard error.

not excessive or deficient with the firm commitment to blood glucose control. Fetal pancreatic $\beta$-cells have been reported to be damaged when mother's energy intake is restricted and can cause impaired glucose tolerance later in life [13]; therefore, it is important that healthcare professionals including physicians, nurses, and nutritionists work together to avoid focusing solely on correcting hyperglycemia and ensure adequate

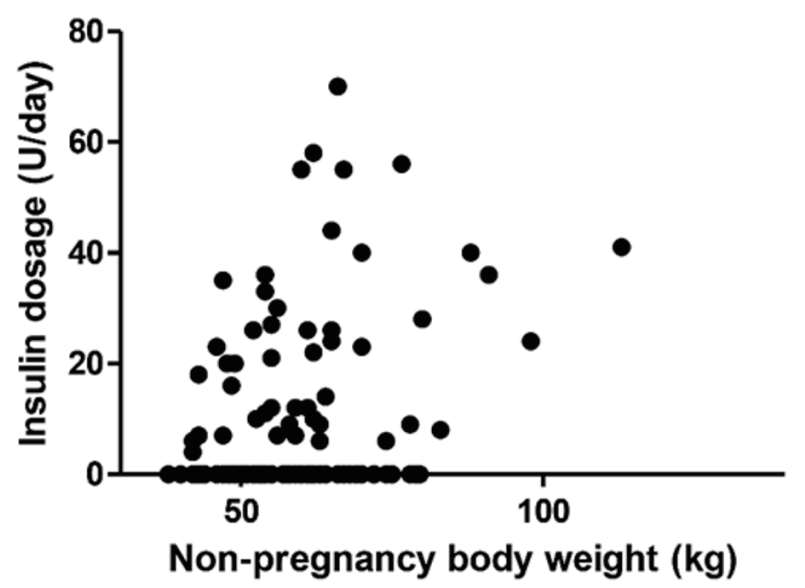

Figure 1. Correlation between non-pregnancy body weight $(\mathrm{kg})$ and insulin dosage (units/day) ( $r=0.360, P=0.012)$. energy intake.

As the second factor, obesity was identified to positively correlate with the insulin dosage. Notably, not the body weight at the time of delivery, but the body weight in the non-pregnant state was a significant positive correlation factor for insulin dosage in this study. Previous studies have shown that mother's obesity while not pregnant and a history of GDM can be risks for GDM [14, 15] and that normal BMI during non-pregnancy reduces the risk for maternal and fetal complications in GDM [16]. These findings suggest that weight control during non-pregnancy can be a preventive measure against complications, particularly in patients with a history of GDM. It has already been reported that the incidence of type 2 diabetes is high in women with a history of GDM [17]. Therefore, women who wish to get pregnant should be encouraged to learn about the means of preventing obesity, such as diet therapy and exercise therapy, and establish a healthy lifestyle to prevent lifestyle diseases. It has also been reported that children born to pregnant women with the diabetes mellitus complication or to those with GDM develop metabolic syndrome and obesity later in life $[18,19]$. Meanwhile, pre-pregnant BMI of $<18.5$ $\mathrm{kg} / \mathrm{m}^{2}$ has been reported to be associated with an increased risk for low-birth-weight infants [20]. Therefore, to prevent GDM and improve the prognosis of mothers and children, it is important to encourage pre-pregnant women to maintain appropriate body weight. 


\section{Acknowledgments}

We would like to thank Enago (www.enago.jp) for English language editing.

\section{Financial Disclosure}

This work was supported by a Grant-in-Aid for Research Activity start-up (17H07056) to HY from the Japan Society for the Promotion of Science.

\section{Conflict of Interest}

The authors declare no conflict of interest.

\section{Informed Consent}

Formal informed consent is not required for this retrospective study.

\section{Author Contributions}

YM analyzed data and wrote the manuscript; HY conceptualized and drafted the manuscript; MY, DS, RS, MA, SF, IH, KT, and $\mathrm{KH}$ collected and analyzed the data.

\section{References}

1. HAPO Study Cooperative Research Group. Hyperglycemia and Adverse Pregnancy Outcome (HAPO) Study: associations with neonatal anthropometrics. Diabetes. 2009;58(2):453-459.

2. Temple RC, Aldridge VJ, Murphy HR. Prepregnancy care and pregnancy outcomes in women with type 1 diabetes. Diabetes Care. 2006;29(8):1744-1749.

3. American Diabetes Association. 12. Management of diabetes in pregnancy. Diabetes Care. 2016;39(Suppl 1):S94-98.

4. HAPO Study Cooperative Research Group, Metzger BE, Lowe LP, Dyer AR, Trimble ER, Chaovarindr U, Coustan DR, et al. Hyperglycemia and adverse pregnancy outcomes. N Engl J Med. 2008;358(19):1991-2002.

5. Kodama Y, Sameshima H, Ohashi M, Ikenoue T. Impact of new gestational diabetes mellitus criteria on stillbirth: a regional population-based study in Japan. J Obstet Gynaecol Res. 2013;39(7):1242-1245.

6. Minakami H, Maeda T, Fujii T, Hamada H, Iitsuka Y, Itakura $\mathrm{A}$, Itoh $\mathrm{H}$, et al. Guidelines for obstetrical practice in Japan: Japan Society of Obstetrics and Gynecology (JSOG) and Japan Association of Obstetricians and Gynecologists (JAOG) 2014 edition. J Obstet Gynaecol Res. 2014;40(6):1469-1499.
7. Japan Diabetes Society. Guidelines for the management of diabetes mellitus 2016. Tokyo; Nankodo. 2016. (Japanese).

8. Ikenoue S, Miyakoshi K, Saisho Y, Sakai K, Kasuga Y, Fukutake M, Izumi Y, et al. Clinical impact of women with gestational diabetes mellitus by the new consensus criteria: two year experience in a single institution in Japan. Endocr J. 2014;61(4):353-358.

9. Ouzounian JG, Rosenheck R, Lee RH, Yedigarova L, Walden CL, Korst LM. One-hour post-glucola results and pre-pregnancy body mass index are associated with the need for insulin therapy in women with gestational diabetes. J Matern Fetal Neonatal Med. 2011;24(5):718-722.

10. National Health and Nutrition Survey Japan, 2016. http:// www.nibiohn.go.jp/eiken/kenkounippon21/en/eiyouchousa/kekka_todoufuken_h28.html.(Japanese). Accessed 5 March 2019.

11. Horie I, Kawasaki E, Sakanaka A, Takashima M, Maeyama M, Ando T, Hanada H, et al. Efficacy of nutrition therapy for glucose intolerance in Japanese women diagnosed with gestational diabetes based on IADPSG criteria during early gestation. Diabetes Res Clin Pract. 2015;107(3):400-406.

12. Reader D, Splett P, Gunderson EP, Diabetes C, Education Dietetic Practice G. Impact of gestational diabetes mellitus nutrition practice guidelines implemented by registered dietitians on pregnancy outcomes. J Am Diet Assoc. 2006;106(9):1426-1433.

13. Fernandez-Twinn DS, Ozanne SE. Mechanisms by which poor early growth programs type-2 diabetes, obesity and the metabolic syndrome. Physiol Behav. 2006;88(3):234243.

14. Black MH, Sacks DA, Xiang AH, Lawrence JM. Clinical outcomes of pregnancies complicated by mild gestational diabetes mellitus differ by combinations of abnormal oral glucose tolerance test values. Diabetes Care. 2010;33(12):2524-2530.

15. Sugiyama T, Nagao K, Metoki H, Nishigori H, Saito M, Tokunaga H, Nagase S, et al. Pregnancy outcomes of gestational diabetes mellitus according to pre-gestational BMI in a retrospective multi-institutional study in Japan. Endocr J. 2014;61(4):373-380.

16. Rogozinska E, Chamillard M, Hitman GA, Khan KS, Thangaratinam $S$. Nutritional manipulation for the primary prevention of gestational diabetes mellitus: a meta-analysis of randomised studies. PLoS One. 2015;10(2):e0115526.

17. Bellamy L, Casas JP, Hingorani AD, Williams D. Type 2 diabetes mellitus after gestational diabetes: a systematic review and meta-analysis. Lancet. 2009;373(9677):17731779.

18. Barker DJ, Osmond C. Infant mortality, childhood nutrition, and ischaemic heart disease in England and Wales. Lancet. 1986;1(8489):1077-1081.

19. Moore TR. Fetal exposure to gestational diabetes contributes to subsequent adult metabolic syndrome. Am J Obstet Gynecol. 2010;202(6):643-649.

20. Misugi Takuya, et al. The effect of maternal weight gain during pregnancy on prenatal prognosis. Adv Obstet Gynecol. 2013;65(3):243-250. 